Pacific Journal of Mathematics

AUTOMORPHISMS AND NONSELFADJOINT CROSSED 


\title{
AUTOMORPHISMS AND NONSELFADJOINT CROSSED PRODUCTS
}

\author{
KICHI-SUKE SAITO
}

\begin{abstract}
We are interested in the invariant subspace structure of the nonselfadjoint crossed product determined by a finite von Neumann algebra $M$ and a trace preserving automorphism $\alpha$. In this paper we investigate the form of two-sided invariant subspaces for the case that $\alpha$ is ergodic on the center of $M$.
\end{abstract}

1. Introduction. In this paper, we consider the typical finite maximal subdiagonal algebras which are called nonselfadjoint crossed products. These algebras are constructed as certain subalgebras of crossed products of finite von Neumann algebras by trace preserving automorphisms. Recently, McAsey, Muhly and the author studied the invariant subspace structure and the maximality of these algebras (cf. [4], [5], [6], [7]).

Let $M$ be a von Neumann algebra with a faithful normal tracial state $\tau$ and let $\alpha$ be ${ }^{*}$-automorphism of $M$ such that $\tau \circ \alpha=\tau$. We regard $M$ as acting on the noncommutative Lebesgue space $L^{2}(M, \tau)$ (cf. [10]) and consider the Hilbert space

$$
L^{2}=\left\{f: Z \longrightarrow L^{2}(M, \tau) \mid \Sigma\|f(n)\|_{2}^{2}<\infty\right\}
$$

which may be identified with $l^{2}(Z) \otimes L^{2}(M, \tau)$. Let $\mathcal{L}$ (resp. $\Re$ ) be the left (resp. right) crossed product of $M$ and $\alpha$, and let $\mathscr{Q}_{+}$(resp. $\Re_{+}$) be the left (resp. right) nonselfadjoint crossed product of $\mathbb{R}$ (resp. $\Re$ ) (cf. §2). In [6], we showed that the following three conditions are equivalent; (i) $M$ is a factor; (ii) a conditioned form of the Beurling-Lax-Halmos theorem is valid; and (iii) $\mathbb{S}_{+}$is a maximal $\sigma$-weakly closed subalgebra of $2 . \quad$ Furthermore, in [7], we proved that $\alpha$ fixes the center $3(M)$ of $M$ elementwise if and only if the Beurling-Lax-Halmos theorem is valid. However, if $\alpha$ does not fix the center $3(M)$ of $M$ elementwise, then the form of invariant subspace is very complicated. Considering the reduction theory with respect to the abelian subalgebra $\{z \in Z(M): \alpha(z)=z\}$ of $3(M)$, it seems to be sufficient to investigate the case that $\alpha$ is ergodic on $3(M)$. Therefore, our aim in this paper is to study the invariant subspace structure of $L^{2}$ when $\alpha$ is ergodic on $3(M)$. We now suppose that $\alpha$ is ergodic on $\mathcal{L}(M)$. Then every two-sided invariant subspace of $L^{2}$ which is not left-reducing is left-pure, left-full, right-pure and right-full (Theorems 3.2 and 4.5). Further, if $\&$ is a factor, then every proper two-sided invariant subspace of $L^{2}$ is of 
the form $\left\{f \in L^{2}: \sum_{k=-\infty}^{n} e_{k} f(n)=f(n), n \in Z\right\}$, where $\left\{e_{n}\right\}_{n=-\infty}^{\infty}$ is a family of mutually orthogonal central projections of $M$ such that $\sum_{n=-\infty}^{\infty} e_{n}=1$ and $\alpha\left(e_{n}\right) \leqq \sum_{k=-\infty}^{n+1} e_{k}$ (Theorems 3.3 and 4.6). However, if $3(M)$ is atomic and there is some $k>0$ such that $\alpha^{k}$ is inner, then we present a two-sided invariant subspace of $L^{2}$ which is not the above form (Example 4.7). In case $M=L^{\infty}(X)$, McAsey in [4] and [5] studied about these results.

In the next section, we define the nonselfadjoint crossed products. In $\S 3$, we consider the case that $3(M)$ is nonatomic. Finally, in $\S 4$, we study two-sided invariant subspace of $L^{2}$ when $3(M)$ is atomic.

The author would like to thank the referee for his valuable suggestions.

2. Preliminaries. We suppose that $M$ is a von Neumann algebra with a faithful normal tracial state $\tau$ and $\alpha$ is a ${ }^{*}$-automorphism of $M$ which preserves $\tau$; i.e., $\tau \circ \alpha=\tau$. Let $L^{2}(M, \tau)$ be the noncommutative Lebesgue space associated with $M$ and $\tau$ in Segal [10]. We denote the operators in the left regular representation of $M$ on $L^{2}(M, \tau)$ by $l_{x}, x \in M$, and those in the right regular representation by $r_{x}$. Put $l(M)=\left\{l_{x}: x \in M\right\}$ and $r(M)=\left\{r_{x}: x \in M\right\}$. Since $\tau \circ \alpha=\tau$, there is a unitary operator $u$ on $L^{2}(M, \tau)$ induced by $\alpha$. To construct a crossed product, we consider the Hilbert space $L^{2}$ defined by

$$
\left\{f: Z \longrightarrow L^{2}(M, \tau) \mid \sum_{n \in Z}\|f(n)\|_{2}^{2}<\infty\right\}
$$

where $\|\cdot\|_{2}^{2}$ is the norm of $L^{2}(M, \tau)$. For $x \in M$, we define operators $L_{x}, R_{x}, L_{o}$ and $R_{o}$ on $L^{2}$ by the formulae $\left(L_{x} f\right)(n)=x f(n),\left(R_{x} f\right)(n)=$ $f(n) \alpha^{n}(x),\left(L_{\delta} f\right)(n)=u f(n-1)$ and $\left(R_{\delta} f\right)(n) f(n-1), g \in L^{2}, n \in Z$. Put $L(M)=\left\{L_{x}: x \in M\right\}$ and $R(M)=\left\{R_{x}: x \in M\right\}$. We set $\mathbb{L}=\left\{L(M), L_{\delta}\right\}^{\prime \prime}$ and $\Re=\left\{R(M), R_{\delta}\right\}^{\prime \prime}$ and define the left (resp. right) nonselfadjoint crossed product $\mathscr{Q}_{+}\left(\operatorname{resp} . \Re_{+}\right)$to be the $\sigma$-weakly closed subalgebra of $\mathcal{L}$ (resp. $\Re$ ) generated by $L(M)$ (resp. $R(M)$ ) and $L_{\delta}$ (resp. $R_{\delta}$ ).

The automorphism group $\left\{\beta_{t}\right\}_{t \in R}$ of $\&$ dual to $\alpha$ in the sense of Takesaki [9] is implemented by the unitary representation of $R$, $\left\{W_{t}\right\}_{t \in R}$, defined by the formula $\left(W_{t} f\right)(n)=e^{2 \pi \text { int }} f(n), f \in L^{2}$; that is, $\beta_{t}(T)=W_{t} T W_{t}^{*}, T \in \mathcal{R}$, by definition. Similarly, we define $\beta_{t}(T)=$ $W_{t} T W_{t}^{*}, T \in \Re$. It is elementary to check that the spectral resolution of $\left\{W_{t}\right\}_{t \in R}$ is given by the formula $W_{t}=\sum_{n=-\infty}^{\infty} e^{2 \pi \text { int }} E_{n}$, where $E_{n}$ is the projection on $L^{2}$ defined by the formula

$$
\left(E_{n} f\right)(k)= \begin{cases}f(n), & k=n, \\ 0, & k \neq n\end{cases}
$$


We also define the integral

$$
\varepsilon_{n}(T)=\int_{0}^{1} e^{-2 \pi i n t} \beta_{t}(T) d t, \quad T \in \mathbb{R} .
$$

Furthermore, we define $H^{2}=\left\{f \in L^{2}: f(n)=0, n<0\right\}$. We refer the reader to [6] and [7] for discussions of these algebras including some of their elementary properties.

Definition 2.1. Let $\mathfrak{M}$ be a closed subspace of $L^{2}$. We say that $\mathfrak{M}$ is: left-invariant, if $\mathfrak{L}_{+} \mathfrak{M} \subseteq \mathfrak{M}$; left-reducing, if $\mathfrak{R M} \subset \mathfrak{M}$; left-pure, if $\bigcap_{n \in Z} L_{\delta}^{n} \mathbb{M}=\{0\}$; and left-full, if $\bigvee_{n \in Z} L_{i}^{n} \mathfrak{M}=L^{2}$. The right hand versions of these concepts are defined similarly, and a closed subspace which is both left and right is called two-sided invariant. If $\mathfrak{M}$ is both left-reducing and right-reducing, $\mathfrak{M}$ is said to be two-sided reducing.

Throughout this paper, we suppose that $\alpha$ is ergodic on the center $3(M)$ of $M$. By the ergodicity of $\alpha$ on $3(M), 3(M)$ is either nonatomic or atomic. Therefore, in $\S \S 3$ and 4 , we consider the invariant subspaces of $L^{2}$ in two cases, respectively.

3. Case $3(M)$ is nonatomic. In this section we investigate the structure of two-sided invariant subspaces of $L^{2}$ for the case when $3(M)$ is nonatomic. To prove this, we need the following lemma. We believe that it is known. But, for completeness, we have included a proof.

Lemma 3.1. $\{L(M), R(M)\}^{\prime}=\left\{L(\mathcal{B}(M)),\left\{E_{n}\right\}_{n=-\infty}^{\infty}\right\}^{\prime \prime}$.

Proof. On $L^{2}$, which we identify with a direct sum of copies of $L^{2}(M, \tau)$, the operators $L_{x}$ and $R_{x}, x \in M$, have these matricial representations;

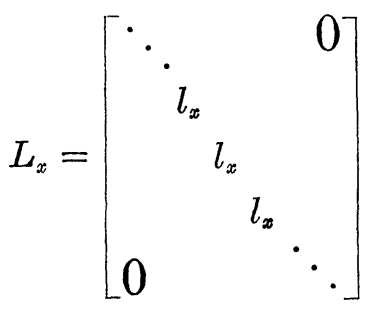




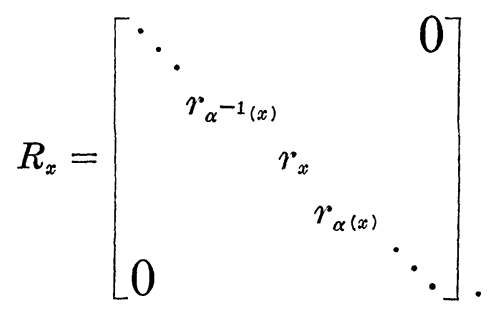

Any operator $A$ in $\{L(M), R(M)\}^{\prime}$ is in $L(M)^{\prime}$ and so has a matricial representation $A=\left[r_{x_{n, m}}\right]$ for suitable $x_{n, m}$ in $M$. In order for $A$ to commute with $R(M)$, it is necessary and sufficient that for each pair $(n, m)$, the equation $\alpha^{n}(y) x_{n, m}=x_{n, m} \alpha^{m}(y)$ holds for all $y$ in $M$. This is equivalent to the validity of the equation

$$
y \alpha^{-n}\left(x_{n, m}\right)=\alpha^{-n}\left(x_{n, m}\right) \alpha^{m-n}(y) \text {, for all } y \text { in } M \text {. }
$$

If $n=m, x_{n, n}$ lies in $3(M)$. Suppose that $n \neq m$ and $x_{n, m} \neq 0$. Let $q$ be the central support projection of $\alpha^{-n}\left(x_{n, m}\right)$. Since $\alpha$ is ergodic on $3(M)$, it is well-known that $\alpha^{n}$ is freely-acting on $3(M)$ for $n \neq 0$. Thus there exists a nonzero projection $p \in 3(M)$ such that $\alpha^{m-n}(p) p=$ 0 and $0<p \leqq q$. By (3.1), $p \alpha^{-n}\left(x_{n, m}\right)=\alpha^{-n}\left(x_{n, m}\right) \alpha^{m-n}(p)=0$. This is a contradiction and so $x_{n, m}=0$. Therefore $\{L(M), R(M)\}^{\prime} \subset\{L(3(M))$, $\left.\left\{E_{n}\right\}_{n=-\infty}^{\infty}\right\}^{\prime \prime}$. The converse is clear. This completes the proof.

By [5, Corollary 4.3], every two-sided invariant subspace which is left- (or right-) reducing is two-sided reducing. Therefore, since $\mathbb{Z}$ is a factor by the ergodicity of $\alpha$ on $3(M)$, such a space is $\{0\}$ or $L^{2}$.

THEOREM 3.2. Every proper two-sided invariant subspace of $L^{2}$ is left-pure, left-full, right-pure and right-full.

Proof. Put $\mathfrak{M}_{1}=\bigcap_{n=1}^{\infty} L_{j}^{n} \mathfrak{M}$ and let $P$ be the projection of $L^{2}$ onto $\mathfrak{M}_{1}$. Since $\mathfrak{M}_{1}$ is left-reducing, $P \in \mathbb{R}^{\prime}=\Re$. Since $\mathfrak{M}_{1}$ is rightinvariant, $R_{\delta} P R_{\delta}^{*} \leqq P$ and $P \in R(M)^{\prime}$. By the finiteness of $\Re, P \in$ $\mathfrak{R} \cap \Re=3(\mathbb{L})$. Since $\mathbb{R}$ is a factor and $P \neq 1, \mathfrak{M}_{1}=\{0\}$. The rest are analogously proved.

Let $\left\{e_{n}\right\}_{n=-\infty}^{\infty}$ be a family of mutually orthogonal central projections in $M$ such that $\sum_{n=-\infty}^{\infty} e_{n}=1$ and $\alpha\left(e_{n}\right) \leqq \sum_{m=-\infty}^{n+1} e_{m}$. Put

$$
L^{2}\left(\left\{e_{n}\right\}_{n=-\infty}^{\infty}\right)=\left\{f \in L^{2}: \sum_{m=-\infty}^{n} e_{m} f(n)=f(n), \text { for all } n\right\} \text {. }
$$

Then it is clear that $L^{2}\left(\left\{e_{n}\right\}_{n=-\infty}^{\infty}\right)$ is a two-sided invariant subspace of $L^{2}$ which is not left-reducing. Conversely, we have the following theorem. 
THeOREM 3.3. Suppose that $\mathrm{Z}(M)$ is nonatomic. Then every proper two-sided invariant subspace of $L^{2}$ is of the form $L^{2}\left(\left\{e_{n}\right\}_{n=-\infty}^{\infty}\right)$ where $\left\{e_{n}\right\}_{n=-\infty}^{\infty}$ is a family of mutually orthogonal central projections in $M$ such that $\sum_{n=-\infty}^{\infty} e_{n}=1$ and $\alpha\left(e_{n}\right) \leqq \sum_{m=-\infty}^{n+1} e_{m}$.

Proof. Let $\mathfrak{M}$ be a proper two-sided invariant subspace of $L^{2}$. By Theorem 3.2, $\mathfrak{M}$ is right-pure. Put $\widetilde{F}=\mathfrak{M} \ominus R_{\delta} \mathfrak{M}$ and let $P$ (resp. $P_{\mathfrak{m}}$ ) be the projection of $L^{2}$ onto $\widetilde{F}$ (resp. $\mathfrak{M}$ ). It is clear that $P \in\{L(M), R(M)\}^{\prime}$. By Lemma 3.1, there is a family $\left\{e_{n}\right\}_{n=-\infty}^{\infty}$ of central projections of $M$ such that $(P f)(n)=e_{n} f(n)$. Thus we have for all $n$,

$$
e_{n} f(0)=e_{n}\left(R_{\dot{\delta}}^{n} f\right)(n)=\left(P R_{\dot{\delta}}^{n} f\right)(n)=\left(R_{\tilde{\delta}}^{*^{n}} P R_{\dot{\delta}}^{n} f\right)(0)
$$

and so, for every $m, n(m \neq n)$,

$$
e_{m} e_{n} f(0)=\left(\left(R_{\delta}^{* m} P R_{\delta}^{m}\right)\left(R_{\tilde{o}}^{*^{n}} P R_{\tilde{o}}^{n}\right) f\right)(0)=0,
$$

because $R_{\delta}^{*^{n}} P R_{\delta}^{n}$ and $R_{i}^{*^{m}} P R_{\dot{\delta}}^{m}$ are orthogonal. This implies that $e_{m} e_{n}=$ $0, m \neq n$. Further, since $\left(R_{\delta}^{k} P R_{\delta}^{* k} f\right)(n)=e_{n-k} f(n)$, for all $k$ and $n$, we have

$$
\left(P_{\mathfrak{m}} f\right)(n)=\left(\left(\sum_{k=0}^{\infty} R_{\dot{o}}^{k} P R_{\dot{o}}^{*^{k}}\right) f\right)(n)=\sum_{k=0}^{\infty} e_{n-k} f(n)=\sum_{k=-\infty}^{n} e_{k} f(n) .
$$

Hence $f \in \mathfrak{M}$ if and only if $f(n)=\sum_{k=-\infty}^{n} e_{k} f(n)$. Now, if $f \in L^{2}$, then

$$
\begin{aligned}
\left(L_{\delta} P_{\text {: }} L_{\delta}^{*} f\right)(n) & =u\left(P_{\text {W. }} L_{\hat{o}}^{*} f\right)(n-1)=u \sum_{k=-\infty}^{n-1} e_{k}\left(L_{\hat{o}}^{*} f\right)(n-1) \\
& =u \sum_{k=-\infty}^{n-1} e_{k} u^{*} f(n)=\sum_{k=-\infty}^{n-1} \alpha\left(e_{k}\right) f(n) .
\end{aligned}
$$

Since $L_{o} \mathfrak{M} \subseteq \mathfrak{M}$, this implies that $\sum_{k=-\infty}^{n-1} \alpha\left(e_{k}\right) \leqq \sum_{k=-\infty}^{n} e_{k}$. Since $\alpha$ is ergodic on $\mathfrak{Z}(\mathfrak{M})$ and $\alpha\left(\sum_{n=-\infty}^{\infty} e_{n}\right) \leqq \sum_{n=-\infty}^{\infty} e_{n}, \sum_{n=-\infty}^{\infty} e_{k}=1$. Therefore $\mathfrak{M}=L^{2}\left(\left\{e_{n}\right\}_{n=-\infty}^{\infty}\right)$. This completes the proof.

4. Case $3(M)$ is atomic. In this section we investigate the structure of two-sided invariant subspaces of $L^{2}$ for the case when $3(M)$ is atomic. We suppose that $\alpha$ is ergodic on $3(M)$ and $3(M)$ is atomic. Since $M$ is finite, there is a family $\left\{p_{n}\right\}_{n=0}^{N-1}$ of mutually orthogonal minimal projections in $3(M)$ such that $\sum_{n=0}^{N-1} p_{n}=1, \alpha\left(p_{n}\right)=$ $p_{n+1}, n=0,1, \cdots, N-2$, and $\alpha\left(p_{N-1}\right)=p_{0}$. Hence $M p_{n}$ is a factor and $\left.\alpha^{k N}\right|_{M p_{n}}$ is a *automorphism of $M p_{n}$. In this section we keep the notations.

To prove Theorems 4.5 and 4.6, we need the following lemmas. As may be well-known, we include them for completenss in our version. At first, we have the following lemma easily and so the proof will be omitted. 
LEMMA 4.1. The following conditions are equivalent.

(i) $\alpha^{k}$ is outer for all $k \neq 0$; and

(ii) for every $n=0,1, \cdots, N-1,\left.\alpha^{k N}\right|_{\mu_{n}}$ is outer for all $k \neq 0$;

(iii) for some $n,\left.\alpha^{k N}\right|_{M p_{n}}$ is outer for all $k \neq 0$.

As in Lemma 3.1, we have the following lemma.

LEMMA 4.2. If $\alpha^{k}$ is outer for all $k \neq 0$, then $\{L(M), R(M)\}^{\prime}=$ $\left\{L(\Re(M)) \cup\left\{E_{n}\right\}_{n=-\infty}^{\infty}\right\}^{\prime \prime}$.

Proof. As in the proof of Lemma 3.1, take $A=\left[r_{x_{n, m}}\right] \in L(M)^{\prime} \cap$ $R(M)^{\prime}$. Then $y \alpha^{-n}\left(x_{n, m}\right)=\alpha^{-n}\left(x_{n, m}\right) \alpha^{m-n}(y), y \in M$. If $n=m, x_{n, n} \in$ $3(M)$ and, if $m-n \neq k N$, then $x_{n, m}=0$. Thus, suppose that $x_{n, m} \neq$ $0, m-n=k N$. Put $z=\alpha^{-k N}\left(x_{n, n+k N}\right)$. Then there is a $j$ such that $z p_{j} \neq 0$ and so $y z=z \alpha^{k N}(y), y \in M p_{j}$. Hence $l_{y} l_{z}=l_{z} v l_{y} v^{*}$, where $v=$ $u^{k N}$, and so $l_{z} v \in l(M)^{\prime}=r(M)$. Since $\left(l_{z} v\right)\left(l_{z} v\right)^{*} \in l(M) \cap r(M)=l(3(M))$, $z z^{*} \in Z(M)$. Hence we have $z z^{*} p_{j}=\left\|z p_{j}\right\|^{2} p_{j}$. If $w$ is then chosen $w=z p_{j} /\left\|z p_{j}\right\|$, then $w$ is a partial isometry which is an element of $M p_{j}$. Since $M p_{j}$ is finite, $w$ is a unitary operator when viewed as an element of $M p_{j}$ and implements $\left.\alpha^{-k N}\right|_{M p_{j}}$. By Lemma 4.1, this is a contradiction and so $z=0$. This completes the proof.

It is well-known that if $M$ is a factor and $\alpha^{\prime \prime}$ is outer for all $k \neq 0$, then $\mathfrak{R}$ is a factor. In this case, the converse is true and we have the following.

LEMMA 4.3. $\quad \alpha^{k}$ is outer for all $k \neq 0$ if and only if $\mathbb{R}$ is a factor.

Proof. ( $\leftarrow)$. If $\alpha^{k N}$ is inner for some $k \neq 0$, then there is a unitary operator $v \in M$ such that $\alpha^{k N}(x)=v x v^{*}$. Thus we have $v \alpha(x) v^{*}=\alpha^{k N+1}(x)=\alpha(v) \alpha(x) \alpha\left(v^{*}\right)$. Hence we have that, for all $n, \alpha^{n}(v)$ and $v$ induce the same automorphism by conjugation. So $L_{\alpha^{n}(v)} L_{x} L_{\alpha^{n}\left(v^{*}\right)}=L_{\alpha^{k N}(x)}$, hence $L_{x} L_{\alpha^{n}\left(v^{*}\right)}=L_{\alpha^{n}\left(v^{*}\right)} L_{\alpha^{k N}(x)}$. From $L_{\delta}^{*} L_{x} L_{\delta}=$ $L_{\alpha^{-1}(x)}, L_{\alpha^{k N(x)}} L_{\hat{o}}^{k N}=L_{\delta}^{k N} L_{x}$. Thus $L_{x} L_{\alpha^{n}\left(v^{*}\right)} L_{\delta}^{k N}=L_{\alpha^{n}\left(v^{*}\right)} L_{\alpha^{k N}(x)} L_{\hat{o}}^{k N}=$ $L_{\alpha^{n}\left(v^{*}\right)} L_{\delta}^{k N} L_{x}$ and $L_{\alpha^{n}\left(v^{*}\right)} L_{\delta}^{k N} \in L(M)^{\prime}$. Since $L_{\delta} L_{x} L_{\delta}^{*}=L_{\alpha(x)}$, for all $x \in$ $M$, we have $L_{\delta}^{k N} L_{x} L_{\delta}^{* k N}=L_{\alpha^{k N}(x)}$. Since $\alpha^{k N}(v)=v$, we have also $\alpha^{k N}\left(\alpha^{n}(v)\right)=\alpha^{n}(v)$ and $\alpha^{k N}\left(\alpha^{n}\left(v^{*}\right)\right)=\alpha^{n}\left(v^{*}\right)$. Hence $L_{\hat{o}}^{k N}$ commutes with $L_{\alpha^{n}(v)}$ and $L_{\alpha^{n}\left(v^{*}\right)}$. Put $w=v \alpha(v) \cdots \alpha^{k N-1}(v)$. Since $\alpha(w)=w$, we have $L_{w^{*}}\left(L_{i}^{k N}\right)^{k N} \in L(M)^{\prime}$. On the other hand, since $\alpha\left(w^{*}\right)=w^{*}, L_{w^{*}}$ commutes with $L_{\delta}$ and $L_{w^{*}}\left(L_{\delta}^{k N}\right)^{k N}$ commutes with $L_{\delta}$. Thus we have $L_{w^{*}}\left(L_{\delta}^{k N}\right)^{k_{N}} \in \mathbb{Z}(\mathbb{R})$. Therefore $\mathbb{Q}$ is not a factor. This completes the proof. 
$(\rightarrow)$. Suppose that $\alpha^{k}$ is outer for all $k \neq 0$. Take $A \in 3(\Omega) \subset$ $L(M)^{\prime} \cap R(M)^{\prime}$. By Lemma 4.2, there is a sequence $\left\{x_{n}\right\} \in 3(M)$ such that $A=\left[l_{x_{n}}\right]$. Since $A$ commutes with $L_{\delta}$ and $R_{\delta}, x_{n}=x_{0}$ and $\alpha\left(x_{0}\right)=$ $x_{0}$. Since $\alpha$ is ergodic, $A=\lambda 1$ for some $\lambda$. Therefore $\mathscr{L}$ is a factor. This completes the proof.

Next we investigate the center of crossed products when $\alpha^{k}$ is inner for some $k \neq 0$.

LEMmA 4.4. Suppose that $\mathbb{Q}$ is not a factor. Then there are a unitary operator $v \in M$ and $k>0$ such that $3(\&)=\left\{L_{v} L_{\delta}^{k N}\right\}^{\prime \prime}$.

Proof. Put $\widetilde{\beta}_{t}=\beta_{t \mid 3(\mathrm{~g})}$. Then $\left\{\widetilde{\beta}_{t}\right\}_{t \in R}$ is a $\sigma$-weakly continuous one-parameter group of *automorphisms of $3(\mathbb{R})$ with period 1 and is ergodic on $3(\Omega)$ in the sense that, if $T \in Z(\Omega)$ such that $\widetilde{\beta}_{t}(T)=$ $T, t \in R$, then $T=\lambda 1$ for some complex number $\lambda$. For every $n \in Z$, put $K_{n}=\left\{T \in \mathbb{Z}(\mathbb{R}): \beta_{t}(T)=e^{2 \pi \mathrm{int}} T, t \in R\right\}$. Then it is clear that $\varepsilon_{n}(3(\Omega))=K_{n}$. Let $Z_{1}=\left\{n \in Z: K_{n} \neq\{0\}\right\}$. We claim that $Z_{1}$ is a subgroup of the additive group $Z$. Let $T_{n}$ be a nonzero element in $K_{n}$ such that $\left\|T_{n}\right\|=1$ for a fixed $n \in Z_{1}$. Then $T_{n}^{*} T_{n}, T_{n} T_{n}^{*}$ is nonzero elements of $K_{0}$ (cf. [9, Lemma $\left.1(\mathrm{a})\right]$ ). Since $\left\{\widetilde{\beta}_{t}\right\}_{t \in R}$ is ergodic on $3(\Omega), T_{n}$ is a unitary operator. By [9, Lemma $\left.1(\mathrm{a})\right]$, we have $K_{n}=C T_{n}$ for every $n \in Z_{1}$. Therefore, $Z_{1}$ is a subgroup of $Z$. Let $m$ be the smallest positive integer in $Z_{1}$. By the group property of $Z_{1}$, we have $Z_{1}=m Z$. Hence, by [9, Lemma 1(a)], $K_{n m}=C T_{m}^{n}$, $n \in Z$. By [9, Theorem 1], $3(\mathbb{R})$ is generated by $T_{m}$. Since $\varepsilon_{m}(\mathbb{Z})=$ $L(M) L_{i}^{m}$ (cf. [3, Corollary 4.3.2]), there is a unitary operator $v$ in $M$ such that $T_{m}=L_{v} L_{i}^{m}$. Since $T_{m} \in Z(\Omega)$, we have, for $x \in M$,

$$
L_{\alpha^{m}(x)}=L_{j}^{m} L_{x} L_{j}^{* m}=L_{v}^{*} T_{m} L_{x}\left(L_{v}^{*} T_{m}\right)^{*}=L_{v^{*}} L_{x} L_{v}=L_{v^{*} x v}
$$

and so $\alpha^{m}$ is inner. Since $\alpha^{n}$ is not inner for all $n \neq j N$, there is a $k>0$ such that $m=k N$. This completes the proof.

The following theorem is proved by McAsey [5] in case $M=$ $l^{\infty}(X),(X)=\left\{x_{0}, x_{1}, \cdots, x_{N-1}\right\}$. We present the simple proof in more general setting.

THEOREM 4.5. Every two-sided invariant subspace which is not left-reducing is left-pure, left-full, right-pure and right-full.

Proof. If $\alpha^{k}$ is outer for all $k \neq 0$, by Lemma $4.3, \&$ is a factor. Then we have this theorem as in the proof of Theorem 3.2. Suppose now that $\mathbb{Q}$ is not a factor. Let $\mathfrak{M}$ be a two-sided invariant subspace which is not left-reducing and let $P$ be the projection of $L^{2}$ onto 
$\bigcap_{n>0} L_{i}^{n} \mathfrak{M}$. Put $B=\{T \in \mathbb{R}: T \mathfrak{M} \subset \mathfrak{M}\} . \quad$ As in the proof of Theorem 3.2 and [6, Theorem 4.1], $P \in B \cap Z(\&)$. Since $3(\mathfrak{Z}) \cap \mathfrak{L}_{+}$is a maximal $\sigma$-weakly closed subalgebra of $3(\Omega)([6$, Theorem 2.3]), we find $3(\Omega) \cap$

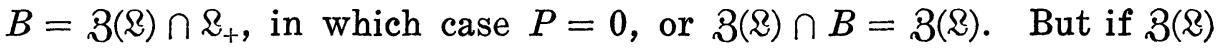
were contained in $B$, by Lemma $4.4, L_{v} L_{i}^{* k N} \in B$ for some unitary $v \in M$ and some $k>0$. Since $\mathfrak{M}$ is left-invariant, $L_{\delta}^{*} \in B$ and so $B=$ 2. This is a contradiction. Therefore we conclude once more $P=0$ and so $\mathfrak{M}$ is left-pure.

Next, let $P$ be the projection of $L^{2}$ onto $\bigcap_{n>0} R_{\delta}^{n} \mathfrak{M}$. Put $B=$ $\{T \in \Re: T \mathfrak{M} \subset \mathfrak{M}\} . \quad$ As before, $P \in \mathbb{Z}(\mathbb{L}) \cap B$ and we find that $3(\Omega) \cap$

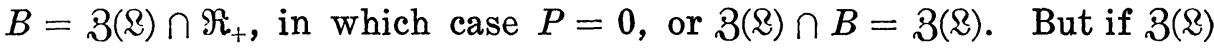
were contained in $B$, then there exists a unitary $v \in M$ and $k>0$ such that $R_{v} R_{i}^{* k N} \in \mathbb{Z}(\mathbb{R})$, as in the proof of Lemma 4.4. Thus $B=$ $\mathfrak{R}$. Therefore $\mathfrak{M}$ is right-reducing. By [6, Corollary 4.3], $\mathfrak{M}$ is twosided reducing. This is a contradiction. The rest is analogously proved. This completes the proof.

As in $\S 2$, we define $L^{2}\left(\left\{e_{n}\right\}_{n=-\infty}^{\infty}\right)=\left\{f \in L^{2}: \sum_{m=-\infty}^{n} e_{m} f(n)=f(n)\right.$, for all $n\}$ for a family $\left\{e_{n}\right\}_{n=-\infty}^{\infty}$ of mutually orthogonal central projections in $M$ such that $\sum_{n=-\infty}^{\infty} e_{n}=1$ and $\alpha\left(e_{n}\right) \leqq \sum_{m=-\infty}^{n+1} e_{m}$. Then it is clear that $L^{2}\left(\left\{e_{n}\right\}_{n=-\infty}^{\infty}\right)$ is a two-sided invariant subspace of $L^{2}$ which is not left-reducing. Observe that all but finitely many of $e_{n}$ are zero. Conversely, we have the following theorem by Lemmas $4.2,4.3$ and Theorem 4.5.

THEOREM 4.6. Suppose that $a^{k}$ is outer for all $k \neq 0$. Then every proper two-sided invariant subspace of $L^{2}$ is of the form $L^{2}\left(\left\{e_{n}\right\}_{n=-\infty}^{\infty}\right)$ where $\left\{e_{n}\right\}_{n=-\infty}^{\infty}$ is a family of mutually orthogonal central projections in $M$ such that $\sum_{n=-\infty}^{\infty} e_{n}=1$ and $\alpha\left(e_{n}\right) \leqq \sum_{m=-\infty}^{n+1} e_{m}$.

Finally, if $\mathbb{Q}$ is not a factor, then Theorem 4.6 is not valid. That is, there is a two-sided invariant subspace of $L^{2}$ which is not of the form $L^{2}\left(\left\{e_{n}\right\}_{n=-\infty}^{\infty}\right)$.

ExAmple 4.7. Suppose that $3(\Omega)=\left\{L_{v} L_{i}^{k N}\right\}^{\prime \prime}$ for some unitary $v$ in $M$ and some $k>0$. Let $\theta$ be a finite Blaschke product with zeros $\left\{\lambda_{1}, \lambda_{2}, \cdots, \lambda_{s}\right\}$ such that $0<\left|\lambda_{j}\right|<1$. This $\theta$ has the form

$$
\prod_{j=1}^{s}\left(\left|\lambda_{j}\right|\left(\lambda_{j}-z\right)\right) /\left(\lambda_{j}\left(1-\bar{\lambda}_{j} z\right)\right) \text {. }
$$

Let $V=\theta\left(L_{v} L_{\delta}^{k N}\right)$ be the unitary operator in $3(\Omega)$ defined by $\theta$ and the operator $L_{v} L_{i}^{k N}$ via the functional calculus. Let $\sum_{i=0}^{\infty} a_{i} z^{i}$ be the power series for $\theta$. Since the power series converges absolutely, the series $\sum_{i=0}^{\infty} a_{i}\left(L_{v} L_{\delta}^{k N}\right)^{i}$ converges in norm to the operator $V$. Observe that $a_{0} \neq 0$ and $V \in \mathfrak{R}_{+}$. Put $\mathfrak{M}=V H^{2}$. It is clear that $\mathfrak{M}$ 
is a two-sided invariant subspace of $H^{2}$ which is not left-reducing. We now suppose that $\mathfrak{M}$ is of the form $L^{2}\left(\left\{e_{n}\right\}_{n=-\infty}^{\infty}\right)$. Since $V f \in \mathfrak{M}$, $f \in H^{2}$, we have $\sum_{n=-\infty}^{m} e_{n}(V f)(m)=(V f)(m),(V f)(-m)=0, m>0$, and

$$
\begin{aligned}
(V f)(0) & =\sum_{n=0}^{\infty} a_{n}\left(\left(L_{v} L_{\dot{o}}^{k N}\right)^{n} f\right)(0)=\sum_{n=0}^{\infty} a_{n} v^{n} u^{n k N} f(-n k N) \\
& =a_{0} f(0) .
\end{aligned}
$$

Thus this implies that $\sum_{m=-\infty}^{0} e_{m}=1$ and $\sum_{m=-\infty}^{-1} e_{m}=0$. Therefore $e_{0}=1$ and $e_{n}=0, n \neq 0$. Hence $\mathfrak{M}=H^{2}$ and so it is clear that $V^{*} \in \mathfrak{Q}_{+}$ which is clearly impossible for $V$ constructed above. Hence $\mathfrak{M} \neq$ $L^{2}\left(\left\{e_{n}\right\}_{n=-\infty}^{\infty}\right)$.

\section{REFERENCES}

1. W. B. Arveson, On groups of automorphisms of operator algebras, J. Functional Anal., 15 (1974), 217-243.

2. H. Behncke, Automorphisms of crossed products, Tôhoku Math. J., 21 (1969), 580-600.

3. R. I. Loebl and P. S. Muhly, Analyticity and flows in von Neumann algebras, J. Functional Anal., 29 (1978), 214-252.

4. M. McAsey, Invariant subspaces of nonselfadjoint crossed products, Pacific J. Math., 96 (1981), 457-473.

5. —_, Canonical models for invariant subspaces, Pacific J. Math., 91 (1980), $377-395$.

6. M. McAsey, P. S. Muhly and K. -S. Saito, Nonselfadjoint crossed products (Invariant subspaces and maximality), Trans. Amer. Math. Soc., 248 (1979), 381-409.

7. —_, Nonselfadjoint crossed products II, J. Math. Soc. Japan, 33 (1981), 485-495.

8. G. K. Pedersen, C*-Algebras and Their Automorphism Groups, Academic Press, 1979.

9. K. -S. Saito, The Hardy spaces associated with a periodic flow on a von Neumann algebra, Tôhoku Math. J., 29 (1977), 69-75.

10. I. E. Segal, A non-commutative extension of abstract integration, Ann. of Math., 57 (1953), 401-457.

11. M. Takesaki, Duality for crossed products and the structure of von Neumann algebras of type III, Acta Math., 131 (1973), 249-310.

12. G. Zeller-Meier, Produit croisés d'une $C^{*}$-algèbre par un groupe d'automorphismes, J. Math. Pures Appl., (9) 47 (1968), 101-239.

Received May 5, 1981 and in revised form October 19, 1981.

NiIgata University

NiIgata, 950-21

JAPAN 



\section{PACIFIC JOURNAL OF MATHEMATICS}

\section{EDITORS}

DONALD BABBITT (Managing Editor)

University of California

Los Angeles, California 90024

HUGO ROSSI

University of Utah

Salt Lake City, UT 84112

C. C. MOORE and ARTHur AGUS

University of California

Berkeley, CA 94720
J. DUGUNDJI

Department of Mathematics

University of Southern California

Los Angeles, California 90007

R. FINN and J. MILGRAM

Stanford University

Stanford, California 94305

\section{ASSOCIATE EDITORS}
R. Arnes
E. F. BECKENBACH
B. H. NeumanN
F. WOLF
K. YösHIDA

\section{SUPPORTING INSTITUTIONS}

UNIVERSITY OF ARIZONA

UNIVERSITY OF BRITISH COLUMBIA

CALIFORNIA INSTITUTE OF TECHNOLOGY

UNIVERSITY OF CALIFORNIA

MONTANA STATE UNIVERSITY

UNIVERSITY OF NEVADA, RENO

NEW MEXICO STATE UNIVERSITY

OREGON STATE UNIVERSITY
UNIVERSITY OF OREGON

UNIVERSITY OF SOUTHERN CALIFORNIA

STANFORD UNIVERSITY

UNIVERSITY OF HAWAII

UNIVERSITY OF TOKYO

UNIVERSITY OF UTAH

WASHINGTON STATE UNIVERSITY

UNIVERSITY OF WASHINGTON 


\section{Pacific Journal of Mathematics}

Vol. 102, No. $1 \quad$ January, 1982

S. Agou, Degré minimum des polynômes $f\left(\sum_{i=0}^{m} a_{i} X^{p^{r i}}\right)$ sur les corps finis

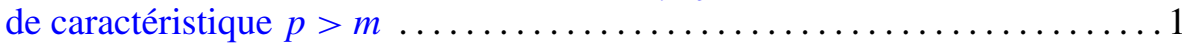

Chi Cheng Chen, On the image of the generalized Gauss map of a complete

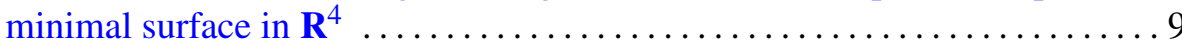

Thomas Curtis Craven and George Leslie Csordas, On the number of real

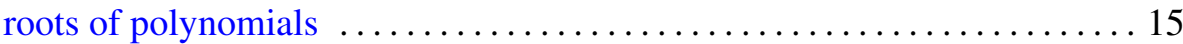

Allan L. Edelson and Kurt Kreith, Nonlinear relationships between oscillation and asymptotic behavior ....................... 29

B. Felzenszwalb and Antonio Giambruno, A commutativity theorem for

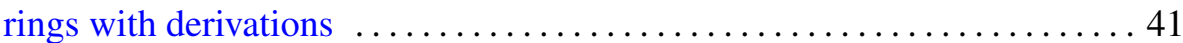

Richard Elam Heisey, Manifolds modelled on the direct limit of lines . . . . . 47

Steve J. Kaplan, Twisting to algebraically slice knots $\ldots \ldots \ldots \ldots \ldots \ldots 5$

Jeffrey C. Lagarias, Best simultaneous Diophantine approximations. II.

Behavior of consecutive best approximations $\ldots \ldots \ldots \ldots \ldots \ldots \ldots 61$

Masahiko Miyamoto, An affirmative answer to Glauberman's conjecture . . . 889

Thomas Bourque Muenzenberger, Raymond Earl Smithson and L. E.

Ward, Characterizations of arboroids and dendritic spaces ........... 107

William Leslie Pardon, The exact sequence of a localization for Witt

groups. II. Numerical invariants of odd-dimensional surgery

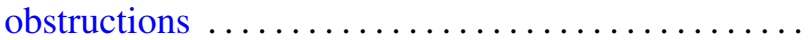

Bruce Eli Sagan, Bijective proofs of certain vector partition identities

Kichi-Suke Saito, Automorphisms and nonselfadjoint crossed products ...

John Joseph Sarraille, Module finiteness of low-dimensional PI rings ...

Gary Roy Spoar, Differentiable curves of cyclic order four . .

William Charles Waterhouse, Automorphisms of quotients of $\Pi \mathrm{GL}\left(n_{i}\right)$

Leslie Wilson, Mapgerms infinitely determined with respect to right-left equivalence

Rahman Mahmoud Younis, Interpolation in strongly logmodular

algebras 